\title{
Multireference calculations of the phosphorescence and photodissociation of chlorobenzene
}

\author{
Ya-Jun Liu \\ Department of Quantum Chemistry, Uppsala University, Box 518, S-751 20 Uppsala, Sweden \\ Petter Persson \\ Department of Quantum Chemistry, Uppsala University, Box 518, S-751 20 Uppsala, Sweden \\ and CALTECH, Beckman Institute 139-74, Materials and Process Simulation Center, Pasadena, \\ California 91125 \\ Sten Lunell ${ }^{\mathrm{a})}$ \\ Department of Quantum Chemistry, Uppsala University, Box 518, S-751 20 Uppsala, Sweden
}

(Received 30 June 2004; accepted 7 September 2004)

\begin{abstract}
Multireference complete active space self-consistent-field (CASSCF) and multireference CASSF second-order perturbation theory (MSCASPT2) calculations were performed on the ground state and a number of low-lying excited singlet and triplet states of chlorobenzene. The dual phosphorescence observed experimentally is clearly explained by the MSCASPT2 potential-energy curves. Experimental findings regarding the dissociation channels of chlorobenzene at 193, 248, and $266 \mathrm{~nm}$ are clarified from extensive theoretical information including all low-energy potential-energy curves. (C) 2004 American Institute of Physics. [DOI: 10.1063/1.1810135]
\end{abstract}

\section{INTRODUCTION}

Aryl halides play an important role in chemical synthesis and are of practical significance to environmental protection. ${ }^{1,2}$ The properties of their excited states have also received attention in both experimental ${ }^{3-6}$ and theoretical ${ }^{7-10}$ studies.

Takemura et al. observed dual phosphorescence of chlorobenzene $(\mathrm{ClBz})$ in a rigid glass solution. ${ }^{11}$ They confirmed that the dual phosphorescence consists of a broad peak with a maximum at about $480 \mathrm{~nm}$ and a somewhat structured peak with a maximum near $400 \mathrm{~nm}$. The two features correspond to short-lived and long-lived emission, respectively. Their observed lifetimes are $3 \pm 0.4$ and $12 \pm 2 \mathrm{~ms}$, respectively. ${ }^{11}$ They assigned the slow phosphorescence to a $S_{0}$ $\leftarrow T\left(\pi, \pi^{*}\right)$ transition, and the predominant fast phosphorescence to a $T\left(\pi, \sigma^{*}\right)$ state. ${ }^{12}$ Nagaoka et al. ${ }^{7}$ studied the lowlying triplet states of $\mathrm{ClBz}$ by Hartree-Fock (HF) calculations, and they assigned the slow phosphorescence to the transition from the first $T-A_{1}$ state to the $S_{0}$ state, and the fast phosphorescence to the transition from the second $T-B_{1}$ state to the $S_{0}$ state. Rubio-Pons et al. ${ }^{13}$ calculated the lifetime of its lowest triplet state using the complete active space self-consistent-field (CASSCF) method. However, they did not provide high-quality potential-energy curves (PECs) for the $\mathrm{ClBz}$ ground and excited states. Here we present PECs of the ground and several low-lying excited singlet and triplet states from multireference CASSCF second-order perturbation theory ${ }^{14}$ (MSCASPT2) calculations. These curves are important for clearly understanding the dual phosphorescence of $\mathrm{ClBz}$. The interpretation of the experimental results in terms of two low-lying triplet states is further supported

\footnotetext{
a) Author to whom correspondence should be addressed. Electronic mail: sten.lunell@kvac.uu.se
}

by the present calculations. This is one of the objectives of this work.

Experimentally, the photodissociation dynamics of $\mathrm{ClBz}$ has been studied at $266 \mathrm{~nm}$ by femtosecond pump-probe spectroscopy $^{5}$ and crossed laser-molecular-beam technique. ${ }^{15}$ Experiments have also been published for excitations by an excimer laser at 248 (Ref. 16) and 193 nm. ${ }^{16-18}$ It was concluded from these experimental studies that the photodissociation of the $\mathrm{C}-\mathrm{Cl}$ bond in $\mathrm{ClBz}$ following a 193-nm excitation takes place through three different dissociation channels with probabilities of similar magnitudes. The first channel was assigned to a direct dissociation or very fast predissociation, the second channel is via vibrationally excited triplet levels, and the third dissociation channel is via highly excited vibrational levels of the ground electronic state (hot molecules). The photodissociation of $\mathrm{ClBz}$ at 248 $\mathrm{nm}$ was proposed to occur dominantly via the second and third of the above-mentioned channels. The photodissociation at $266 \mathrm{~nm}$ has been given alternative explanations. On one hand, it was proposed to be due to a hot molecule mechanism by Wang et al. ${ }^{15}$ On the other hand, Kadi et al. ${ }^{5}$ assigned it to the decay of an initially excited $\left(\pi, \pi^{*}\right)$ state to a repulsive triplet $\left(n, \sigma^{*}\right)$ state due to spin-orbit coupling, and they observed its time constant to be $1 \mathrm{~ns}^{5}$. The experimental explanation of the photodissociation channels of $\mathrm{ClBz}$ must thus, in many ways, still be regarded as tentative. For example, we still do not know if the first channel at 193 $\mathrm{nm}$ is due to direct dissociation or fast predissociation, or which specific states contribute to the photodissociation at the longer wavelengths.

High-quality calculated PECs can provide much useful information for a detailed understanding of the dissociation dynamics following excitation at a specific wavelength. The main objective of this paper is therefore to provide accurate 
calculated PECs to enable a clear and detailed explanation of the photodissociation mechanism of $\mathrm{ClBz}$ at different wavelengths. Besides our recent theoretical study of the aryl halide photodissociations at $266 \mathrm{~nm},{ }^{10}$ Nagaoka et al. calculated the PECs of some low-lying triplet states of $\mathrm{ClBz}$ by the HF method. ${ }^{7}$ We have not found any calculated PECs of $\mathrm{ClBz}$ pertinent to the photodissociation experiments conducted at 248 and $193 \mathrm{~nm}$, although it has been suggested that accurate calculations of higher excited electronic states could be useful to clarify experimental findings for ClBz. ${ }^{19}$ Investigating a larger number of excited states allows us to consider changes in the photodissociation behavior for initial photoexcitations using higher-energy photons, adding another dimension to our recent aryl halide studies that focused on substitution effects for the low-energy photodissociation channels. ${ }^{9,10}$ In these works, the MSCASPT2 method was used to calculate vertical excitation energies $\left(T_{v}\right)$, and PECs of ground and excited states.

This paper is organized in such a way that we first describe the methods and results of the quantum-chemical calculations before broadening the discussion to comparisons with experimental observations and previous theoretical calculations on the phosphorescence and photodissociation of ClBz.

\section{COMPUTATIONAL METHODS}

Most calculations were performed using the MOLCAS 5.4 quantum-chemistry software. ${ }^{20}$ Atomic natural orbital (ANO) basis sets ${ }^{21}$ were used for all the atoms. $\mathrm{C}(10 s 6 p 3 d)$, $\mathrm{H}(7 s 3 p)$, and $\mathrm{Cl}(13 s 10 p 4 d)$ are contracted to $(3 s 2 p 1 d)$, $(2 s 1 p)$, and $(4 s 3 p 2 d)$, respectively. The second-order Moller-Plesset perturbation theory (MP2) (Ref. 22) method with the $6-311+\mathrm{G}(d, p)$ basis $\operatorname{set}^{23}$ was used to calculate basis set superposition error (BSSE) corrected energies. These calculations were carried out using the GAUSSIAN 03 program. ${ }^{24}$ The results have been checked for BSSE, which was found to be of negligible importance in this case.

The geometries of the ground and excited states were optimized using the CASSCF method. MSCASPT2 methods were then used to calculate the vertical excitation energies $\left(T_{v}\right)$ of the three lowest-lying singlet and triplet states of every irreducible representation in the $C_{2 v}$ symmetry of the molecule. That is to say, 12 singlet and 12 triplet states were calculated. Of course, these states are not necessarily the 24 overall lowest-lying states. The PECs along the reaction coordinate described by the $\mathrm{Cl}-\mathrm{C}_{6} \mathrm{H}_{5}$ bond distance were calculated using the MSCASPT2 method for all these 24 states. The harmonic vibrational frequencies of the ground state were calculated using the CASSCF method. The same threshold and shift were used in the calculations of all the PECs. In the CASSCF calculations, 12 electrons were active. The active space included the six $p$ orbitals of the phenyl ring, the $\sigma$ and $\sigma^{*}$ orbitals of the $\mathrm{C}_{1}-\mathrm{Cl}$ bond, and the two remaining $3 p$ orbitals of chlorine. The states average technique was used in the CASSCF $T_{v}$ calculations. The $1 s$ cores of carbon and chlorine were frozen in the CASPT2 calculations.
TABLE I. Calculated and experimental ground-state geometry of ClBz.

\begin{tabular}{cccccc}
\hline \hline Method & CASSCF $^{\mathrm{a}}$ & CASSCF $^{\mathrm{b}}$ & RHF $^{\mathrm{c}}$ & UHF $^{\mathrm{d}}$ & Expt. $^{\mathrm{e}}$ \\
\hline$R\left(\mathrm{Cl}-\mathrm{C}_{1}\right)$ & 1.746 & 1.778 & 1.745 & 1.820 & 1.741 \\
$R\left(\mathrm{C}_{1}-\mathrm{C}_{2}\right)$ & 1.403 & 1.392 & 1.383 & 1.374 & 1.393 \\
$R\left(\mathrm{C}_{2}-\mathrm{C}_{3}\right)$ & 1.399 & 1.395 & 1.385 & 1.385 & 1.396 \\
$R\left(\mathrm{C}_{3}-\mathrm{C}_{4}\right)$ & 1.399 & 1.387 & 1.385 & 1.384 & 1.401 \\
\hline \hline
\end{tabular}

${ }^{\mathrm{a}}$ The present paper. $\quad{ }^{\mathrm{d}}$ Reference 7.

${ }^{\mathrm{b}}$ Reference $10 . \quad$ Reference 25.

${ }^{\mathrm{c}}$ Reference 15 .

\section{RESULTS}

\section{A. Ground state}

We used the CASSCF method to optimize the groundstate geometry of ClBz. The present result, the previous reported calculated results, ${ }^{7,10,15}$ and the corresponding experimental fitted values ${ }^{25}$ are listed in Table I. We also optimized the ground-state geometry of ClBz by CASSCF in Ref. 10 . Due to the use of an effective core potential basis set on the $\mathrm{Cl}$ atom in Ref. 10, the optimized $\mathrm{Cl}-\mathrm{C}_{1}$ bond distance $(1.778 \AA)$ differs a little more from the experimental fitted value $(1.742 \AA)$. $\mathrm{Cl}$ is not a very heavy atom, so we used an ANO basis set here to get a better optimized geometry. From Table I, the CASSCF optimized ground-state geometry is seen to agree very well with the corresponding experimental fitted values.

The frequencies of the $\mathrm{ClBz}$ ground state have previously been calculated by the Becke-3-Lee-Yang-Parr, MP2, and CASSCF methods, ${ }^{26}$ and the HF method. ${ }^{27}$ However, as the symmetries of the vibrational modes were not indicated in Ref. 26, we only compare the present CASSCF calculated result with the HF results in Ref. 27 and the experimental results; see Table II. The present CASSCF calculated vibrational ground-state frequencies agree well with the experiment both in magnitude and in the assignment of the vibrational modes. ${ }^{28-30}$

\section{B. Vertical excitation energies}

The MSCASPT2 method was used to calculate the vertical excitation energy, $T_{v}$, of the three lowest-lying singlet and triplet states of every irreducible representation in the $C_{2 v}$ symmetry of $\mathrm{ClBz}$ at its CASSCF optimized groundstate geometry. From the calculated results, we determined the seven lowest singlet and six lowest triplet states of $\mathrm{ClBz}$ to be $S_{0}-A_{1}, S_{1}-B_{2}, S_{2}-A_{1}, S_{3}-B_{2}, S_{4}-B_{1}, S_{5}-A_{2}$, and $S_{6}-A_{1}$, and $T_{1}-A_{1}, T_{2}-B_{2}, T_{3}-A_{1}, T_{4}-B_{2}, T_{5}-B_{1}$, and $T_{6}-A_{1}$, respectively; see Table III. For ease of notations, the other calculated five singlet and six triplet states are also denoted using consecutive subscript numbers in Table III, although these numbers may not necessarily reflect the actual order of the higher excited states. Due to the use of different basis sets and a better optimized ground-state geometry, the $T_{v}$ values and order in the present calculation are a little different from those in Ref. 10. The errors in CASPT2 computed excitation energies are typically less than $0.3 \mathrm{eV}^{31}$ Double-resonance multiphoton ionization photoelectron spectroscopy ${ }^{19}$ indicated that the first five singlet excited states of $\mathrm{ClBz}$ are $S-A_{1}, S-B_{2}, S-A_{1}, S-B_{2}$, and $S-A_{1}$, 
TABLE II. The calculated and experimental frequencies $\left(\right.$ in $\mathrm{cm}^{-1}$ ) of $\mathrm{ClBz}$ ground state.

\begin{tabular}{cccccc}
\hline \hline Mode & Symmetry & CASSCF & CASSCF $^{\mathrm{a}}$ & HF $^{\mathrm{a}, \mathrm{b}}$ & Expt. \\
\hline 1 & $a_{1}$ & 3381 & 3009 & 3030 & $3082^{\mathrm{c}}$ \\
2 & $a_{1}$ & 3348 & 2980 & 3016 & $3054^{\mathrm{c}}$ \\
3 & $a_{1}$ & 3335 & 2968 & 2994 & $3031^{\mathrm{c}}$ \\
4 & $a_{1}$ & 1714 & 1525 & 1594 & $1586^{\mathrm{d}}$ \\
5 & $a_{1}$ & 1595 & 1420 & 1473 & $1482^{\mathrm{d}}$ \\
6 & $a_{1}$ & 1238 & 1102 & 1154 & $1153^{\mathrm{d}}$ \\
7 & $a_{1}$ & 1145 & 1019 & 1071 & $1093^{\mathrm{d}}$ \\
8 & $a_{1}$ & 1079 & 960 & 999 & $1026^{\mathrm{d}}$ \\
9 & $a_{1}$ & 1047 & 932 & 970 & $1004^{\mathrm{d}}$ \\
10 & $a_{1}$ & 735 & 654 & 681 & $707^{\mathrm{d}}$ \\
11 & $a_{1}$ & 435 & 387 & 361 & $417^{\mathrm{d}}$ \\
12 & $a_{2}$ & 989 & 880 & 980 & $962^{\mathrm{e}}$ \\
13 & $a_{2}$ & 784 & 698 & 970 & $831^{\mathrm{e}}$ \\
14 & $a_{2}$ & 428 & 381 & 407 & $403^{\mathrm{e}}$ \\
15 & $b_{1}$ & 1029 & 916 & 1002 & $981^{\mathrm{e}}$ \\
16 & $b_{1}$ & 888 & 790 & 922 & $903^{\mathrm{e}}$ \\
17 & $b_{1}$ & 772 & 687 & 748 & $741^{\mathrm{e}}$ \\
18 & $b_{1}$ & 721 & 642 & 679 & $684^{\mathrm{e}}$ \\
19 & $b_{1}$ & 510 & 454 & 470 & $467^{\mathrm{e}}$ \\
20 & $b_{1}$ & 204 & 182 & 187 & $197^{\mathrm{e}}$ \\
21 & $b_{2}$ & 3384 & 3012 & 3027 & $3096^{\mathrm{c}}$ \\
22 & $b_{2}$ & 3355 & 2986 & 3004 & $3067^{\mathrm{c}}$ \\
23 & $b_{2}$ & 1668 & 1485 & 1590 & $1598^{\mathrm{d}}$ \\
24 & $b_{2}$ & 1545 & 1375 & 1435 & $1447^{\mathrm{d}}$ \\
25 & $b_{2}$ & 1428 & 1271 & 1303 & $1327^{\mathrm{d}}$ \\
26 & $b_{2}$ & 1253 & 1115 & 1184 & $1272^{\mathrm{d}}$ \\
27 & $b_{2}$ & 1155 & 1028 & 1077 & $1167^{\mathrm{d}}$ \\
28 & $b_{2}$ & 1055 & 939 & 1049 & $1068^{\mathrm{d}}$ \\
29 & $b_{2}$ & 616 & 548 & 601 & $615^{\mathrm{d}}$ \\
30 & $b_{2}$ & 307 & 273 & 286 & $295^{\mathrm{d}}$ \\
\hline \hline
\end{tabular}

${ }^{a}$ Multiplied by 0.89 as recommended in W. J. Hehre, L. Radom, P. v. R. Schleyer, and J. A. Pople, Ab Initio Molecular Orbital Theory (Wiley, New York, 1986).

${ }^{\mathrm{b}}$ Reference 27.

${ }^{\mathrm{c}}$ Reference 28.

${ }^{\mathrm{d}}$ Reference 29.

${ }^{\mathrm{e}}$ Reference 30.

respectively. The first four calculated singlet states agree with the experimental symmetry assignments. The fifth singlet state, however, is a $S-B_{2}$ state according to our calculations, in contrast to the $S-A_{1}$ experimental assignment. The calculated $T_{v}$ values of the $S_{1}-B_{2}, S_{2}-A_{1}$, and $S_{6}-A_{1}$ and the $T_{1}-A_{1}$ excited states agree with the experimental values within the expected accuracy; see Table III. The discrepancies for the $S_{3}-B_{2}, S_{4}-B_{1}$, and $S_{5}-A_{2}$ and the $T_{2}-B_{2}$ excited states are somewhat larger than anticipated. This situation was not improved when we used a bigger active space and a bigger basis set including Rydberg basis, for which we see no obvious explanation. The transition character of every excited state was indicated by the present calculations and also listed in Table III.

\section{CASPT2 potential-energy curves}

High-quality PECs can help to provide a detailed understanding of phosphorescence and photodissociation mechanisms. This includes in particular the Franck-Condon region, which is of particular importance for much of the initial dynamics following photoexcitation. In order to study the changes in geometry of the phenyl ring for the excited states
TABLE III. The MSCASPT2 vertical excitation energies (in eV) and the transition characters of the lowest singlet and triplet excited states of ClBz.

\begin{tabular}{|c|c|c|c|c|}
\hline \multicolumn{3}{|c|}{ MSCASPT2 } & \multicolumn{2}{|c|}{ Expt. $^{\mathrm{a}}$} \\
\hline State $^{b}$ & $T_{v}$ & Transition & State & $T_{v}$ \\
\hline$S_{0}-A_{1}$ & 0.00 & & $S_{0}-A_{1}$ & 0.00 \\
\hline$S_{1}-B_{2}$ & 4.50 & $\left(\pi, \pi^{*}\right)$ & $S_{1}-B_{2}$ & 4.59 \\
\hline$S_{2}-A_{1}$ & 5.70 & $\left(\pi, \pi^{*}\right)$ & $S_{2}-A_{1}$ & 5.77 \\
\hline$S_{3}-B_{2}$ & 6.17 & $\left(\pi, \pi^{*}\right)$ & $S_{3}-B_{2}$ & 6.83 \\
\hline$S_{4}-B_{1}$ & 6.59 & $\left(n, \sigma^{*}\right)$ & $S_{4}-A_{1}$ & 7.02 \\
\hline$S_{5}-A_{2}$ & 7.19 & $\left(n, \pi^{*}\right)$ & $S_{5}$ & 7.58 \\
\hline$S_{6}-A_{1}$ & 7.50 & $\left(\pi, \pi^{*}\right)$ & $S_{6}$ & 7.71 \\
\hline$S_{7}-A_{2}$ & 7.53 & $\left(\pi, \sigma^{*}\right)$ & & \\
\hline$S_{8}-B_{2}$ & 7.53 & $\left(\pi, \pi^{*}\right)$ & & \\
\hline$S_{9}-B_{1}$ & 7.79 & $\left(\pi, \sigma^{*}\right)$ & & \\
\hline$S_{10^{-}} A_{2}$ & 7.86 & $\left(\sigma, \pi^{*}\right)$ & & \\
\hline$S_{11}-B_{1}$ & 8.98 & $\left(n, \sigma^{*}\right)$ & & \\
\hline$T_{1}-A_{1}$ & 3.75 & $\left(\pi, \pi^{*}\right)$ & $T_{1}$ & 3.44 \\
\hline$T_{2}-B_{2}$ & 4.29 & $\left(\pi, \pi^{*}\right)$ & $T_{2}$ & 3.54 \\
\hline$T_{3}-A_{1}$ & 4.37 & $\left(\pi, \pi^{*}\right)$ & & \\
\hline$T_{4}-B_{2}$ & 5.06 & $\left(\pi, \pi^{*}\right)$ & & \\
\hline$T_{5}-B_{1}$ & 6.24 & $\left(n, \sigma^{*}\right)$ & & \\
\hline$T_{6}-A_{1}$ & 6.75 & $\left(\pi, \pi^{*}\right)$ & & \\
\hline$T_{7}-B_{2}$ & 6.81 & $\left(\pi, \pi^{*}\right)$ & & \\
\hline$T_{8}-A_{2}$ & 7.17 & $\left(n, \pi^{*}\right)$ & & \\
\hline$T_{9}-A_{2}$ & 7.46 & $\left(\pi, \sigma^{*}\right)$ & & \\
\hline$T_{10}-A_{2}$ & 7.63 & $\left(\sigma, \pi^{*}\right)$ & & \\
\hline$T_{11}-B_{1}$ & 7.64 & $\left(\pi, \sigma^{*}\right)$ & & \\
\hline$T_{12}-B_{1}$ & 8.34 & $\left(n, \sigma^{*}\right)$ & & \\
\hline
\end{tabular}

${ }^{\mathrm{a}}$ Reference 19 .

${ }^{b}$ According to the present calculations, $S_{0}$ to $S_{6}$ are the first seven singlet states and $T_{1}$ to $T_{6}$ are the first six triplet states, respectively. The following five singlet and six triplet states have been labeled with consecutive subscript numbers for convenience.

compared to the ground state, we optimized the geometries of several excited states of importance to the photodissociation processes (see Sec. IV). The geometries of $S_{1}-B_{2}$, $S_{3}-B_{2}, T_{1}-A_{1}$, and $T_{2}-B_{2}$ excited states were all optimized using the CASSCF method with the $\mathrm{Cl}-\mathrm{C}_{1}$ bond fixed at $1.746 \AA$ which is the CASSCF optimized $\mathrm{Cl}-\mathrm{C}_{1}$ bond distance of the ground state; see Table I. The optimized phenyl geometries of the ground and excited states are compared in Table IV. The ring geometries of both the singlet and the triplet excited states are similar to the $S_{0}-A_{1}$ ground-state geometry. We also calculated the MSCASPT2 energies of these partially optimized excited states and compared these results to the vertical MSCASPT2 excitation energies, $T_{v}$,

TABLE IV. Comparison of CASSCF partially optimized phenyl geometries of the ground and excited states at the ground-state equilibrium $R\left(\mathrm{Cl}-\mathrm{C}_{1}\right)$ bond distance (1.746 ̊).

\begin{tabular}{llllll}
\hline \hline & $S_{0}-A_{1}$ & $S_{1}-B_{2}$ & $S_{3}-B_{2}$ & $T_{1}-A_{1}$ & $T_{2}-B_{2}$ \\
\hline$R\left(\mathrm{C}_{1}-\mathrm{C}_{2}\right)(\AA)$ & 1.403 & 1.433 & 1.421 & 1.467 & 1.428 \\
$R\left(\mathrm{C}_{2}-\mathrm{C}_{3}\right)(\AA)$ & 1.399 & 1.437 & 1.525 & 1.362 & 1.437 \\
$R\left(\mathrm{C}_{3}-\mathrm{C}_{4}\right)(\AA)$ & 1.399 & 1.435 & 1.431 & 1.470 & 1.426 \\
Energy gap $(\mathrm{eV})^{\mathrm{a}}$ & 0.00 & 4.30 & 6.17 & 3.48 & 4.09 \\
Energy gap $(\mathrm{eV})^{\mathrm{b}}$ & 0.00 & 4.50 & 6.35 & 3.75 & 4.29 \\
\hline \hline
\end{tabular}

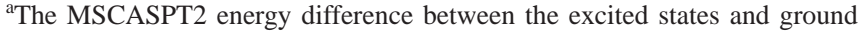
state at their CASSCF optimized geometries.

${ }^{\mathrm{b}}$ The MSCASPT2 vertical excitation energy of excited states at the CASSCF optimized ground-state geometry. 


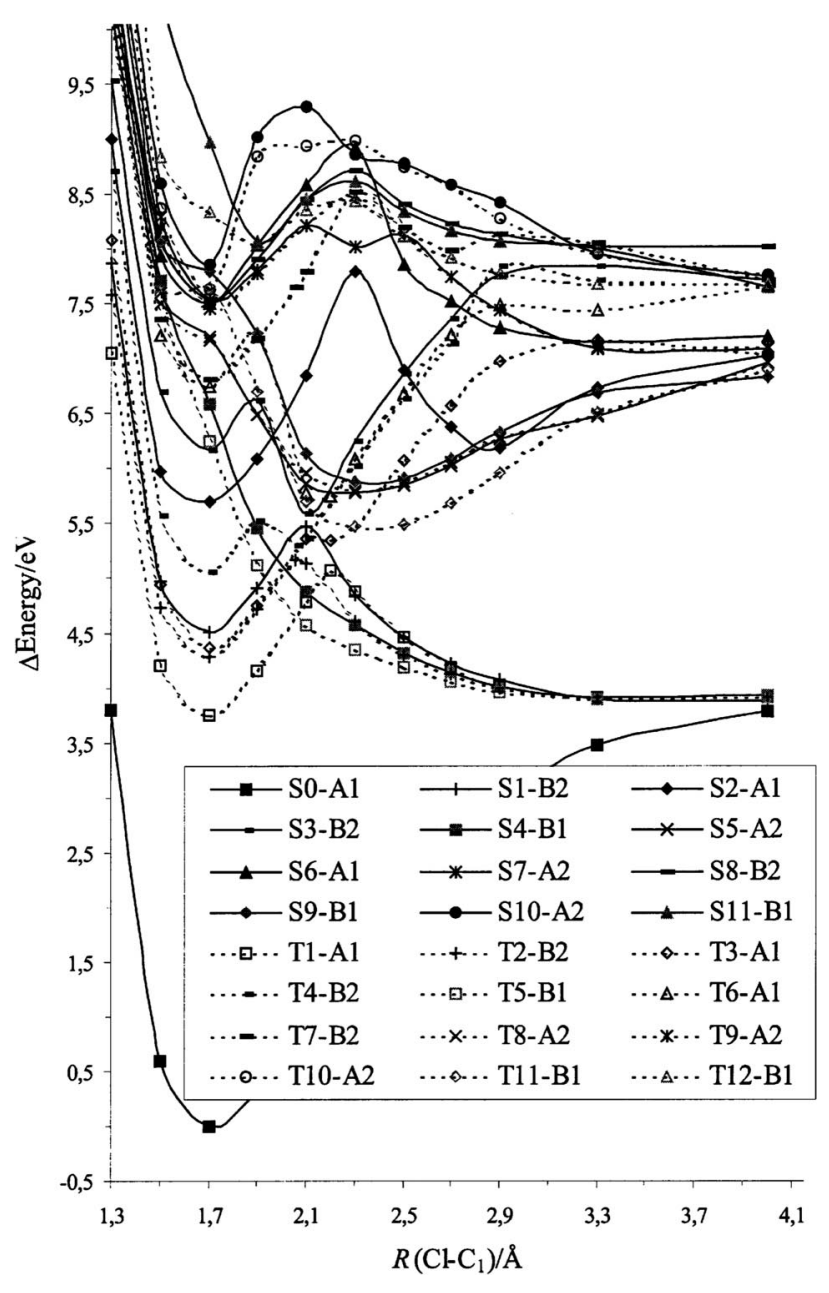

FIG. 1. MSCASPT2 adiabatic potential-energy curves along the $\mathrm{Cl}-\mathrm{C}_{6} \mathrm{H}_{5}$ bond distance of 24 states of chlorobenzene. The dashed lines are used for triplet states and the solid lines are for singlet states.

for the corresponding states as calculated at the ground-state equilibrium geometry in Table IV. The resulting energy differences are less than $0.27 \mathrm{eV}$, which is less than the estimated CASPT2 error of $0.3 \mathrm{eV}{ }^{31}$ In agreement with Refs. 8 and 10 , we thus conclude that optimizations of the excited states only lead to minor changes in both geometries and energies for the excited states. Moreover, optimizing the geometry of every point on the PEC of every excited state is very expensive at this high level of theory. In fact, it is nearly impossible for highly excited states. We therefore fixed the $\mathrm{Cl}-\mathrm{C}_{1}$ bond distance in steps of $0.2 \AA$ from 1.346 to $4.046 \AA$ and partially optimized the geometry of every point on the ground-state PEC by the CASSCF method. These geometries were subsequently used to calculate the MSCASPT2 energies of every point on the PECs of the ground and excited states, yielding MSCASPT2 PECs of the 24 states along the dissociation coordinate given by the $\mathrm{C}_{6} \mathrm{H}_{5}-\mathrm{Cl}$ bond; see Fig. 1. The labels of all the states in Fig. 1 correspond to their $T_{v}$ values in Table III.

\section{DISCUSSION}

\section{A. The dual phosphorescence}

In order to explain the $\mathrm{ClBz}$ phosphorescence, we focus on the PECs of the ground state and the low-lying triplet

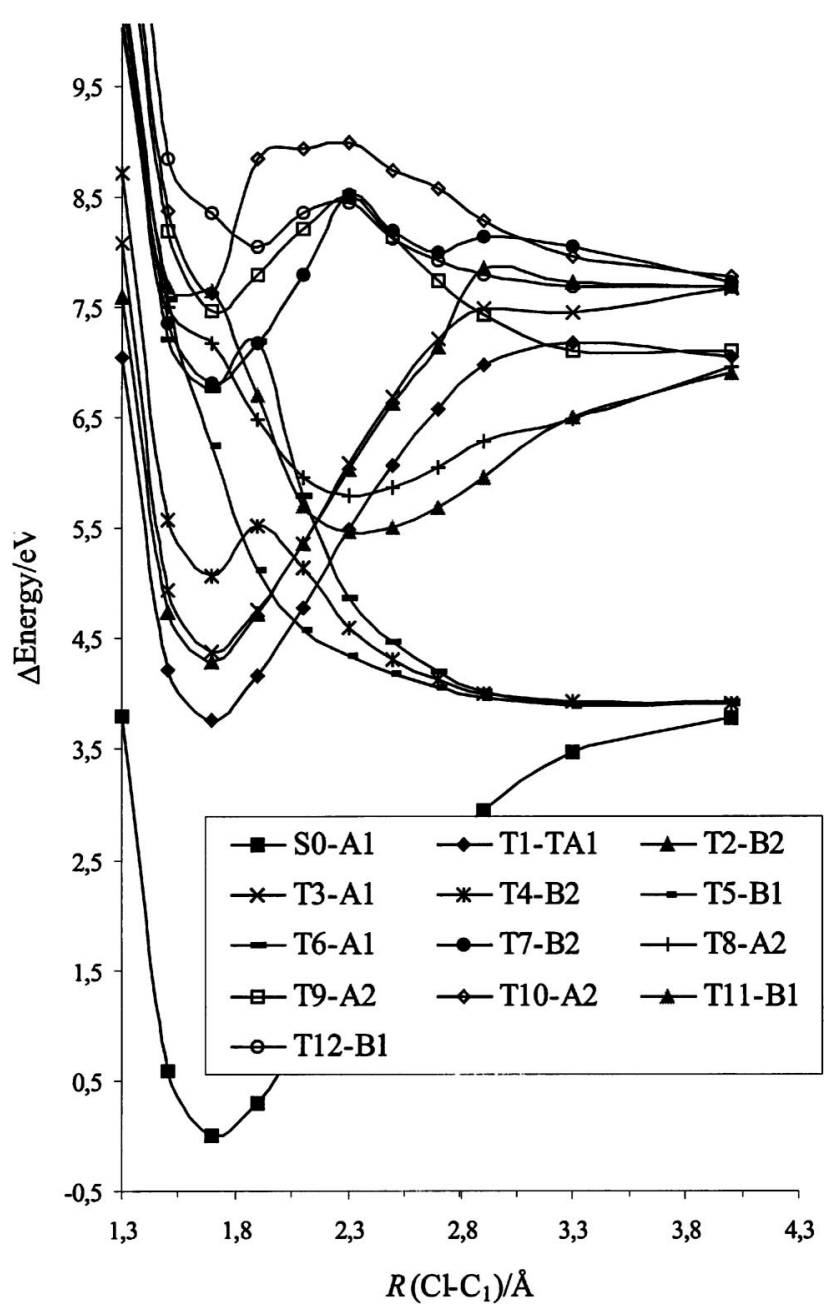

FIG. 2. MSCASPT2 diabatic potential-energy curves along the $\mathrm{Cl}-\mathrm{C}_{6} \mathrm{H}_{5}$ bond distance of the singlet ground state and 12 triplet states of chlorobenzene.

states. In Fig. 1, there is an avoided crossing between the $T_{1}-A_{1}$ and $T_{3}-A_{1}$ states, the $T_{3}-A_{1}$ and $T_{6}-A_{1}$ states, as well as an avoided crossing between the $T_{2}-B_{2}$ and $T_{4}-B_{2}$ states. MSCASPT2 is one of the best methods currently available for calculating accurate potential-energy surfaces, ${ }^{32}$ and the results can be used to diabatize the PECs of low-lying adiabatic states. To investigate the phosphorescence, we extracted the PECs of the ground and all the triplet states from Fig. 1 and drew them diabatically in Fig. 2. From Fig. 2, all the triplet states are bound or quasibound states except the $T_{5}-B_{1}$ state.

We optimized the geometries of $T_{1}-A_{1}, T_{2}-B_{2}$, and $T_{11}-B_{1}$ fully by the CASSCF method; see Table V. From Fig. 2 and Table V, the CASSCF optimized geometries of the $T_{1}-A_{1}, T_{3}-A_{1}, T_{2}-B_{2}$, and $T_{4}-B_{2}$ states are similar to that of the ground state. However, the CASSCF geometry of the $T_{11}-B_{1}$ state has a $\mathrm{Cl}-\mathrm{C}_{1}$ bond distance of $2.577 \AA$, which is $0.822 \AA$ longer than that of the ground state. We also used MSCASPT2 to calculate the transition energies $\left(T_{\mathrm{e}}\right)$ from the $T_{1}-A_{1}, T_{2}-B_{2}, T_{3}-A_{1}, T_{4}-B_{2}$, and $T_{11}-B_{1}$ states to the $S_{0}$ state; see Table VI. The $T_{\mathrm{e}}$ values of the $T_{1}-A_{1}$ and $T_{3}-A_{1}$ were calculated at the CASSCF optimized geometry of the $T_{1}-A_{1}$ state; see Table V. The $T_{\mathrm{e}}$ values of the $T_{2}-B_{2}$ and 
TABLE V. The calculated geometries of selected excited states (in angstrom).

\begin{tabular}{|c|c|c|c|c|c|c|c|c|c|c|}
\hline \multirow[b]{2}{*}{ Method } & \multicolumn{3}{|c|}{$S_{1}-B_{2}$} & \multirow{2}{*}{$\begin{array}{l}S_{2}-A_{1} \\
\mathrm{CAS}^{\mathrm{a}}\end{array}$} & \multirow{2}{*}{$\begin{array}{l}S_{3}-B_{2} \\
\mathrm{CAS}^{\mathrm{a}}\end{array}$} & \multicolumn{2}{|c|}{$T_{1}-A_{1}$} & \multirow{2}{*}{$\begin{array}{l}T_{2}-B_{2} \\
\mathrm{CAS}^{\mathrm{a}}\end{array}$} & \multicolumn{2}{|c|}{$T_{11}-B_{1}$} \\
\hline & $\mathrm{CAS}^{\mathrm{a}}$ & $\mathrm{CIS}^{\mathrm{b}}$ & $\mathrm{HF}^{\mathrm{c}}$ & & & $\mathrm{CAS}^{\mathrm{a}}$ & $\mathrm{HF}^{\mathrm{d}}$ & & $\mathrm{CAS}^{\mathrm{a}}$ & $\mathrm{HF}^{\mathrm{d}}$ \\
\hline$R\left(\mathrm{Cl}-\mathrm{C}_{1}\right)$ & 1.754 & 1.719 & 1.724 & 1.714 & 1.757 & 1.745 & 1.828 & 1.754 & 2.577 & 2.385 \\
\hline$R\left(\mathrm{C}_{1}-\mathrm{C}_{2}\right)$ & 1.433 & 1.412 & 1.411 & 1.506 & 1.421 & 1.467 & 1.375 & 1.427 & 1.437 & 1.443 \\
\hline$R\left(\mathrm{C}_{2}-\mathrm{C}_{3}\right)$ & 1.437 & 1.414 & 1.413 & 1.387 & 1.525 & 1.362 & 1.533 & 1.437 & 1.380 & 1.370 \\
\hline$R\left(\mathrm{C}_{3}-\mathrm{C}_{4}\right)$ & 1.435 & 1.413 & 1.412 & 1.499 & 1.431 & 1.470 & 1.386 & 1.426 & 1.420 & 1.416 \\
\hline
\end{tabular}

$T_{4}-B_{2}$ states were obtained at the CASSCF optimized geometry of $T_{2}-B_{2}$ state, and the $T_{11}-B_{1}$ state $T_{\mathrm{e}}$ value calculated at its CASSCF geometry are given in Table V.

The first triplet state, $T_{1}-A_{1}$, is a bound $\left(\pi, \pi^{*}\right)$ state, and it is likely to be responsible for the slow component of the dual phosphorescence as its calculated $T_{\mathrm{e}}$ value corresponds well to the experimentally observed position of the slow phosphorescence $;{ }^{11}$ see Table VI. This conclusion is the same as the HF calculated result in Ref. 7, and it agrees with the experimental prediction. ${ }^{11}$ As stated in Ref. 7, the second triplet state, $T_{2}-B_{2}$, is also a $\left(\pi, \pi^{*}\right)$ rather than a $\left(\pi, \sigma^{*}\right)$ state. Moreover, according to the present calculations all the first four triplet states are $\left(\pi, \pi^{*}\right)$ states; see Table III.

The first bound $\left(\pi, \sigma^{*}\right)$ state included in Fig. 2 and Table III is $T_{11}-B_{1}$. As mentioned above, it has a calculated $\mathrm{Cl}-\mathrm{C}_{1}$ bond distance of $2.577 \AA$, which is $0.822 \AA$ longer than that of $T_{1}-A_{1}$ state. This difference is about $0.6 \AA$ in the HF calculation; see Table V. The present calculated $T_{\mathrm{e}}$ value from the $T_{11}-B_{1}$ state to the $S_{0}$ state is $2.68 \mathrm{eV}$, which is very close to the experimentally observed position of $480 \mathrm{~nm}$ $(2.58 \mathrm{eV}){ }^{11}$ see Table VI. Therefore, we assign the $T_{11}-B_{1}$ state to be responsible for the fast part of the dual phosphorescence. ${ }^{11}$ The $T_{11}-B_{1}$ state is the second $T$ - $B_{1}$ state and is the same state that HF calculations assigned to the fast phosphorescence. ${ }^{7}$

\section{B. Photodissociation channels of the low-lying excited states}

The shortest excitation wavelength employed in the experiments was $193 \mathrm{~nm}(6.42 \mathrm{eV})$, and we therefore focus mainly on the excited singlet states with $T_{v}$ under or near $193 \mathrm{~nm}$ and the repulsive triplet states which are likely to interact with these singlet states. These are the $S_{0}-A_{1}$,

TABLE VI. The MSCASPT2 transition energies of several triplet states to $S_{0}$ state.

\begin{tabular}{ccc}
\hline \hline Transition & $\begin{array}{c}\text { MSCASPT2 }^{\mathrm{a}} \\
(\mathrm{eV})\end{array}$ & $\begin{array}{c}\text { The dual phosphorescence positions } \\
\text { experimentally observed }\end{array}$ \\
\hline$S_{0} \leftarrow T_{1}-A_{1}$ & 3.10 & $\approx 400 \mathrm{~nm}(3.10 \mathrm{eV})$ \\
$S_{0} \leftarrow T_{2}-B_{2}$ & 4.04 & \\
$S_{0} \leftarrow T_{3}-A_{1}$ & 4.68 & \\
$S_{0} \leftarrow T_{4}-B_{2}$ & 4.96 & $\approx 480 \mathrm{~nm}(2.58 \mathrm{eV})$ \\
$S_{0} \leftarrow T_{11}-B_{1}$ & 2.68 & \\
\hline \hline
\end{tabular}

${ }^{\text {a The }} T_{v}$ value of the $T_{3}-A_{1}$ state was calculated at the CASSCF geometry of the $T_{1}-A_{1}$ state, and the $T_{v}$ value of the $T_{4}-B_{2}$ state was calculated at the CASSCF geometry of the $T_{2}-B_{2}$ state.

${ }^{\mathrm{b}}$ Reference 11 .
$S_{1}-B_{2}, S_{2}-A_{1}, S_{3}-B_{2}, S_{4}-B_{1}$, and $T_{5}-B_{1}$ states. There is one avoided crossing between $S_{1}-B_{2}$ and $S_{3}-B_{2}$. We extracted the PECs of these states from Fig. 1 and drew them diabatically; see Fig. 3. From Fig. 3 and Table III, $S_{1}, S_{2}$, and $S_{3}$ are bound $\left(\pi, \pi^{*}\right)$ states, whereas $S_{4}$ and $T_{5}$ are repulsive $\left(n, \sigma^{*}\right)$ states.

$S_{4}-B_{1}$, with $6.59-\mathrm{eV} T_{v}$, is the highest singlet excited state that the photon of $193-\mathrm{nm}(6.42 \mathrm{eV})$ wavelength used experimentally ${ }^{16-18}$ could reach. It is a repulsive state, which will dissociate directly on a very short time scale. ${ }^{34}$ This direct dissociation should contribute to the fastest of the three channels observed in Ref. 16. The CASSCF optimized geometries of the first three singlet excited states were listed in Table V. As shown in Fig. 3, $S_{3}-B_{2}$ is a quasibound state with a barrier that blocks immediate dissociation. The energy gap between its saddle point and the minimum of the ground state is about $6.6 \mathrm{eV}$. It is possible that molecules excited with photons with a wavelength of $193 \mathrm{~nm}$ can overcome the predissociation barrier if the MSCASPT2 error of $0.3 \mathrm{eV}$ is taken into account. If so, its lifetime will depend on the tunneling rate. This process would proceed as a so-called vibrational or Herzberg's type II predissociation..$^{35}$ This fast predissociation could then also contribute to the fastest dissociation channel as proposed in Ref. 16. From Fig. 3, the PEC of $S_{3}-B_{2}$ crosses the repulsive $\left(n, \sigma^{*}\right) T_{5}-B_{1}$ state. The $\mathrm{Cl}-\mathrm{C}_{1}$ bond distance at the crossing point is about $1.82 \AA$. It is also about $0.2 \mathrm{eV}$ lower than $193 \mathrm{~nm}$. So, we assign the second experimentally observed channel as the intersystem crossing from the bound $\left(\pi, \pi^{*}\right) S_{3}-B_{2}$ state to the repulsive $\left(n, \sigma^{*}\right) T_{5}-B_{1}$ state. The complex will ultimately decay with a rate that depends on the coupling between the two electronic states. This is an electronic predissociation process, which is also called a Herzberg's type I predissociation. ${ }^{35}$ The third photodissociation channel is slower than the first two channels ${ }^{16}$ and was suggested to take place via internal conversion to highly excited vibrational levels of the ground state (hot molecule). ${ }^{16}$ From Fig. 3, it appears likely that the $S_{3}-B_{2}$ state first undergoes internal conversion to the $S_{1}-B_{2}$ state. The lowest vibrational state of $S_{1}$ is much closer in energy to the dissociation limit of $S_{0}$, and thereby makes international conversion from $S_{1}$ to $S_{0}^{*}$ more likely. This mechanism is compatible with the third observed photodissociation channel with lower rate.

The highest singlet excited state the photon with 248-nm $(5.00 \mathrm{eV})^{16}$ wavelength can reach is $S_{1}-B_{2}$, whose $T_{v}$ is 4.50 eV. $S_{1}$ is a bound $\left(\pi, \pi^{*}\right)$ state, which cannot dissociate by itself. However, the $S_{1}-B_{2}$ state can undergo an intersystem 


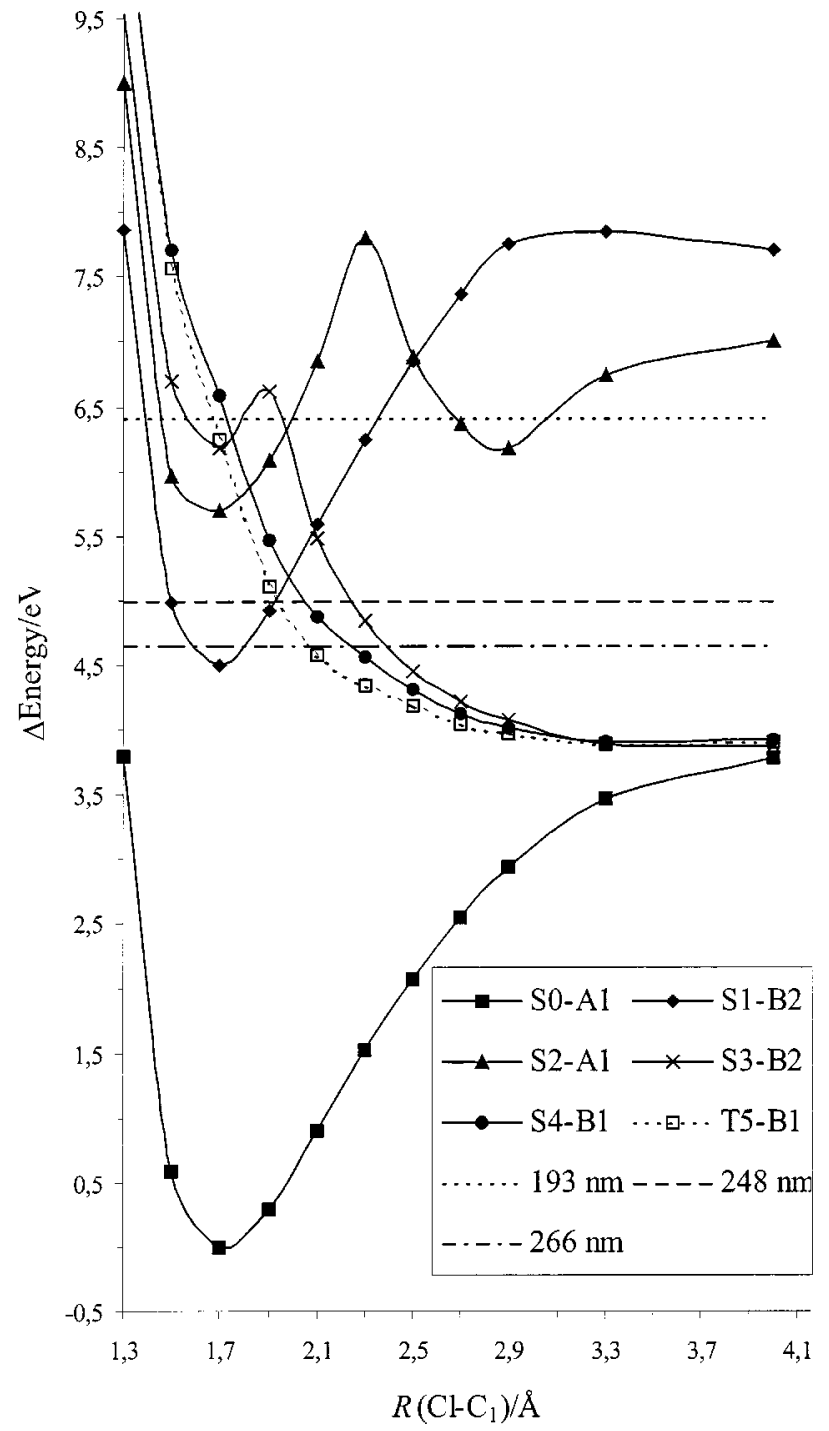

FIG. 3. MSCASPT2 diabatic potential-energy curves along the $\mathrm{Cl}-\mathrm{C}_{6} \mathrm{H}_{5}$ bond distance of one triplet and five singlet states of chlorobenzene. The horizontal dashed lines indicate the 193-, 248-, and 266-nm excitation energy used in previous experiments.

crossing to the repulsive $\left(n, \pi^{*}\right) T_{5}-B_{1}$ state. The $\mathrm{Cl}-\mathrm{C}_{1}$ calculated bond distance at the crossing point is about $2.02 \AA$. The energy gap between this point and the minimum of the ground state is about $5.0 \mathrm{eV}$. The complex will ultimately decay due to spin-orbit coupling between the two electronic states. So, the first channel of photodissociation of $\mathrm{ClBz}$ at $248 \mathrm{~nm}$ is the intersystem crossing from $S_{1}-B_{2}$ to $T_{5}-B_{1}$, and it is a Herzberg's type I predissociation. ${ }^{35}$ From Fig. 3, the PEC of $S_{1}-B_{2}$ also undergoes an internal conversion with the repulsive $\left(n, \pi^{*}\right) S_{4}-B_{1}$ state. The $\mathrm{Cl}-\mathrm{C}_{1}$ bond distance at the crossing point is about $2.06 \AA$. The energy gap between this point and the minimum of the ground state is about $5.15 \mathrm{eV}$. If the 248-nm-wavelength photon used in the experiment overcomes this energy barrier, then the complex will ultimately decay by internal conversion. So, the first channel of photodissociation of $\mathrm{ClBz}$ at $248 \mathrm{~nm}$ is also possibly via the internal conversion from $S_{1}-B_{2}$ to $S_{4}-B_{1}$. The second slower photodissociation channel observed experimentally is again possibly dissociation via the highly vibra- tional levels of the ground state, $S_{0}^{*}$. As discussed above, $S_{0}^{*}$ could be produced by internal conversion from the $S_{1}$ state.

The 266-nm (4.66 eV) -wavelength photon can also reach the $S_{1}-B_{2}$ state. As with the 248-nm excitation, this state cannot dissociate by itself. The PEC of $S_{1}-B_{2}$ crosses to the PECs of $T_{5}-B_{1}$ and $S_{4}-B_{1}$ states. The photoexcitation energies required to reach these two crossing points are about 5.0 and $5.25 \mathrm{eV}$, respectively. The 266-nm (4.66 eV) -wavelength photon is unlikely to reach either of the two points, even considering an estimated error of $0.3 \mathrm{eV}$, just as we said before. ${ }^{10}$ If the predissociation channels are out of reach, the only remaining photodissociation channel at 266 $\mathrm{nm}$ is again via the highly vibrational levels of the ground state, $S_{0}^{*}$, produced by the internal conversion from the $S_{1}$ state, as discussed above. Both Refs. 15 and 5 indicated that the photodissociation following 266-nm excitation is slow, even though the latter assigned it to the decay of an initially excited $\left(\pi, \pi^{*}\right)$ state to a repulsive triplet $\left(n, \sigma^{*}\right)$ state due to spin-orbit coupling.

\section{CONCLUSIONS}

In order to explain the dual phosphorescence and the photodissociation mechanism of chlorobenzene at 193, 248, and $266 \mathrm{~nm}$, we calculated MSCASPT2 potential-energy curves of 24 singlet and triplet states. The bound $\left(\pi, \pi^{*}\right)$ $T_{1}-A_{1}$ state is responsible for the slow part of the dual phosphorescence, and the bound $\left(\pi, \sigma^{*}\right) T_{11}-B_{1}$ state will be responsible for the fast part of the dual phosphorescence. The photodissociation of chlorobenzene at $193 \mathrm{~nm}$ has three channels: (1) a direct dissociation of the repulsive $\left(n, \sigma^{*}\right) S_{4}-B_{1}$ state, or Herzberg's type II predissociation as the quasibound $\left(\pi, \pi^{*}\right) S_{3}-B_{2}$ state dissociates by tunneling, (2) Herzberg's type I predissociation arising from an intersystem crossing from the quasibound $\left(\pi, \pi^{*}\right) S_{3}-B_{2}$ state to the repulsive $\left(n, \sigma^{*}\right) T_{5}-B_{1}$ state, and (3) via highly vibrational levels of the ground state, which are produced from $S_{1}-B_{2}$ state by internal conversion after its internal converting with the $S_{3}-B_{2}$ state. For chlorobenzene photodissociation at $248 \mathrm{~nm}$, there are two channels: (1) Herzberg's type I predissociation arising from an intersystem crossing from quasibound $\left(\pi, \pi^{*}\right) S_{1}-B_{2}$ state to repulsive $\left(n, \sigma^{*}\right) T_{5}-B_{1}$ state, or internal conversion from $\left(\pi, \pi^{*}\right) S_{1}-B_{2}$ state to repulsive $\left(n, \sigma^{*}\right) S_{4}-B_{1}$ state, and (2) via highly vibrational levels of the ground state, which are produced from $S_{1}-B_{2}$ state by internal conversion. However, the photodissociation of chlorobenzene at $266 \mathrm{~nm}$ has only one channel, which is via highly excited vibrational levels of the ground electronic state.

\section{ACKNOWLEDGMENTS}

The Swedish Research Council (VR) and the Magnus Bergvall Foundation are gratefully acknowledged for financial support. The Swedish National Supercomputer Center (NSC) is acknowledged for generous grants of computer resources. The authors wish to thank Dr. Chi-Kung Ni and Professor KeLi Han for helpful discussions about the photodissociation channels. 
${ }^{1}$ T. Matsuura and K. Omura, Bull. Chem. Soc. Jpn. 39, 944 (1996).

${ }^{2}$ J. S. Zhang, T. T. Miau, and Y. T. Lee, in XIVth International Symposium on Molecular Beams (Lawrence Berkely Lab, 1992), pp. 29-31.

${ }^{3}$ P. Y. Cheng, D. Zhong, and A. H. Zewail, Chem. Phys. Lett. 237, 399 (1995).

${ }^{4}$ T. Okutsu, T. Kageyama, N. Kounose, J. Tsuchiya, and H. Hiratsuka, Chem. Phys. Lett. 299, 597 (1999).

${ }^{5}$ M. Kadi, A. N. Tarnovsky, M. Rasmusson, J. Davidsson, and E. Åkesson, Chem. Phys. Lett. 350, 93 (2001).

${ }^{6}$ H. Zhang, R.-S. Zhu, G.-J. Wang, K.-L. Han, G.-Z. He, and N.-Q. Lou, J. Chem. Phys. 110, 2929 (1999).

${ }^{7}$ S. Nagaoka, T. Takemura, H. Baba, N. Koga, and K. Morokuma, J. Phys. Chem. 90, 759 (1986).

${ }^{8}$ M. Rasmusson, R. Lindh, N. Lascoux, A. N. Tarnovsky, M. Kadi, O. Kuhn, V. Sundström, and E. Åkesson, Chem. Phys. Lett. 367, 759 (2003).

${ }^{9}$ Y.-J. Liu, P. Persson, H. O. Karlsson, S. Lunell, M. Kadi, D. Karlsson, and J. Davidsson, J. Chem. Phys. 120, 6502 (2004).

${ }^{10}$ Y.-J. Liu, P. Persson, and S. Lunell, J. Phys. Chem. 108, 2339 (2004).

${ }^{11}$ T. Takemura, Y. Yamada, and H. Baba, Chem. Phys. 68, 171 (1982).

${ }^{12}$ T. Takemura, Y. Yamada, M. Sugawara, and H. Baba, J. Phys. Chem. 90, 2324 (1986).

${ }^{13}$ O. Rubio-Pons, O. Loboda, B. Minaev, B. Schimmelpfenning, O. Vahtras, and H. Agren, Mol. Phys. 101, 2103 (2003).

${ }^{14}$ J. Finley, P.-A. Malmqvist, B. O. Roos, and L. Serrano-Andrés, Chem. Phys. Lett. 288, 299 (1998).

${ }^{15}$ G.-J. Wang, R.-S. Zhu, H. Zhang, K.-L. Han, G.-Z. He, and N.-Q. Lou, Chem. Phys. Lett. 288, 429 (1998).

${ }^{16}$ T. Ichimura, Y. Mori, H. Shinohara, and N. Nishi, Chem. Phys. 189, 117 (1994).

${ }^{17}$ T. Ichimura, Y. Mori, H. Shinohara, and N. Nishi, Chem. Phys. Lett. 122, 51 (1985).

${ }^{18}$ A. Freedman, S. C. Yang, M. Kawasaki, and R. Bersohn, J. Chem. Phys. 72, 1028 (1980).
${ }^{19}$ P. Asselin, F. Piuzzi, M. Mons, and I. Dimicoli, Chem. Phys. 191, 261 (1995).

${ }^{20} \mathrm{~K}$. Andersson, M. Barysz, A. Bernhardsson et al., MOLCAs version 5.4 (University of Lund, Sweden, 2000).

${ }^{21}$ K. Pierloot, B. Dumez, P.-O. Widmark, and B. O. Roos, Theor. Chim. Acta 90, 87 (1995).

${ }^{22}$ M. Head-Gordon, J. A. Pople, and M. J. Frisch, Chem. Phys. Lett. 153, 503 (1988).

${ }^{23}$ W. J. Hehre, L. Radomr, P. V. R. Schleyer, and J. A. Pople, Ab Initio Molecular Orbital Theory (Wiley, New York, 1986).

${ }^{24}$ M. J. Frisch, G. W. Trucks, H. B. Schlegel et al., GAUSSIAN 03, Revision A.1, Gaussian, Inc, Pittsburgh PA, 2003.

${ }^{25}$ S. Cradock, J. M. Muir, and D. W. H. Rankin, J. Mol. Struct. 220, 205 (1990).

${ }^{26}$ P. Imhof and K. Kleinermanns, Chem. Phys. Lett. 270, 227 (2001).

${ }^{27}$ T. G. Wright, S. I. Panov, and T. A. Miller, J. Chem. Phys. 102, 4793 (1995).

${ }^{28}$ D. H. Whiffen, J. Chem. Soc. 1350 (1956)

${ }^{29}$ H. D. Bist, V. N. Sarin, A. Ojha, and Y. S. Jain, Spectrochim. Acta, Part A 26, 841 (1970).

${ }^{30}$ Y. S. Jain and H. D. Bist, J. Mol. Spectrosc. 47, 126 (1973).

${ }^{31}$ M. Merchan, L. Serrano-Andres, M. P. Fülscher, and B. O. Roos, in Recent Advances in Multireference Theory, edited by K. Hirao (World Scientific, Singapore, 1999), Vol. IV, pp. 161-196.

${ }^{32}$ H. Nakamura and D. G. Truhlar, J. Chem. Phys. 118, 6816 (2003).

${ }^{33}$ D. M. Upadhyay, M. K. Shukla, and P. C. Mishra, J. Mol. Struct.: THEOCHEM 531, 249 (2000).

${ }^{34}$ R. Schinke, Photodissociation Dynamics (Cambridge University Press, Cambridge, 1993).

${ }^{35}$ G. Herzberg, Spectra of Polyatomic Molecules, Molecular Spectra and Molecular Structure III (Van Nostrand, New York, 1967). 